\title{
Thyroid disruptor 1,1,1-trichloro-2,2-bis(p-chlorophenyl) ethane (DDT) prevents internalization of TSH receptor
}

\author{
S. Picchietti • M. Belardinelli • A. R. Taddei • \\ A. M. Fausto • M. Pellegrino • R. Maggio $・$ M. Rossi • \\ F. Giorgi
}

Received: 5 September 2008 /Accepted: 19 December 2008 / Published online: 13 February 2009

(C) Springer-Verlag 2009

\begin{abstract}
The thyroid-stimulating hormone (TSH) receptor (TSHr) was made specifically fluorescent by insertion of a tetracysteine motif (TSHr-FlAsH) into the C-terminal end and transiently transfected into COS-7 and HeLa cells. The observation that TSH administration caused the intracellular level of cAMP to increase in both TSHr-FlAsH-transfected cell types indicated that the FlAsH binding motif did not alter normal TSHr functioning. When transfected into HeLa cells and stimulated with TSH, the TSHr-FlAsH receptor exhibited a pronounced perinuclear labelling pattern, whereas labelling remained on the cell surface following pre-incubation with 1,1,1-trichloro-2,2-bis(p-chlorophenyl) ethane (DDT). Chinese hamster ovary (CHO)-TSHr cells probed with anti-TSHr antibodies were fluorescent mainly in the proximity of the plasma membrane, with fluorescence being primarily restricted to a juxta-nuclear position when exposed to $10 \mathrm{mU} / \mathrm{ml} \mathrm{TSH}$ for 1 or $5 \mathrm{~min}$. However, in the presence of DDT, the anti-TSHr fluorescence maintained a peripheral location along the cell plasma membrane, even if CHO-TSHr cells were stimulated with TSH for 1 and $5 \mathrm{~min}$. To verify that DDT acted specifically
\end{abstract}

\author{
S. Picchietti $\cdot$ M. Belardinelli $\cdot$ A. R. Taddei $\cdot$ A. M. Fausto \\ Department of Environmental Sciences, Tuscia University, \\ Viterbo, Italy \\ M. Pellegrino \\ Department of Human Physiology, University of Pisa, \\ Pisa, Italy \\ R. Maggio \\ Department of Experimental Medicine, University of L'Aquila, \\ L'Aquila, Italy \\ M. Rossi $\cdot$ F. Giorgi $(\bowtie)$ \\ Department of Neuroscience, University of Pisa, \\ 56120 Pisa, Italy \\ e-mail: giorgif@biomed.unipi.it
}

on the TSHr, CHO cells transfected with the $\mathrm{A}_{2}$ a receptor were used as controls. Following a 1-min stimulation with 5'-(N-ethyl-carboxamido)-adenosine, $\mathrm{A}_{2}$ a receptors were gradually internalized regardless of the presence of DDT in the culture medium. Finally, immunoelectron microscopy of CHO-TSHr cells showed that a 1-min exposure to TSH sufficed to displace anti-TSHr antibodies tagged with 10$\mathrm{nm}$ gold particles into coated pits and vesicles but that their superficial location was retained along the plasma membrane in the presence of DDT.

Keywords Thyroid-stimulating hormone receptor . Endocytosis $\cdot 1,1,1$-Trichloro-2,2-bis(p-chlorophenyl) ethane $\cdot$ A2a receptor Chinese hamster ovary cells

\section{Introduction}

Much of what is presently known about the mechanisms controlling cell proliferation and differentiation stems from the knowledge that cells can express a number of surface receptors capable of interacting with the external milieu. Depending on the nature of the ligand-receptor interaction, cells may respond specifically to a variety of signals carried by many of the information-coding molecules present in the extracellular environment (Maggio et al. 2007). About 80\% of the hormones, neurotransmitters and growth factors present in the bloodstream of eukaryotic organisms controls a plethora of metabolic responses by interacting specifically with G-protein-coupled receptors (GPCRs) (Bockaert and Pin 1999). Within the large family of the GPCRs, the glycoprotein hormone receptors specific for luteinizing hormone, follicle-stimulating hormone and thyroid-stimulating hormone (TSH) share the common characteristics of having a long ectodomain comprised of numerous leucine 